\title{
Lesson of the month 1: A cough that doesn't fit the mould
}

\author{
Authors: Christina Baggott, ${ }^{A}$ Charles Sharp, ${ }^{B}$ Nidhi Bhatt, ${ }^{C}$ Martin Plummeridge ${ }^{\mathrm{D}}$ and Huzaifa Adamali ${ }^{\mathrm{E}}$
}

Aspergillus disease has a wide spectrum of manifestations within the lungs; however invasive Aspergillus is most commonly associated with immunocompromise or preexisting respiratory disease. Here we present a case of Aspergillus tracheobronchitis causing right middle lobe collapse, masquerading as late-onset asthma in a patient with no pre-existing risk factors following massive inhalation of Aspergillus spores from working with compost. This case highlights the importance of having a high index of suspicion for Aspergillus-related disease even in those with no traditional risk factors.

KEYWORDS: Aspergillus tracheobronchitis, immunocompetent, invasive Aspergillus, spore inhalation

\section{Case report}

A 67-year-old man was referred to respiratory outpatients with a nine-month history of breathlessness, wheeze and cough productive of light-brown tenacious sputum plugs. He had no significant past medical history. In the previous spring, while turning a pile of dry compost he had aspirated a significant amount of the resultant dust. That evening he became breathlessness, however this resolved after a few hours.

The following week, while moving a pile of decaying weeds to the compost, he once again became breathless. This recurred a month later after disposing of weeds into the compost again, with an additional cough on this occasion. His symptoms now persisted, with progressive dyspnoea, cough and occasional wheeze.

Following pulmonary function tests and initial review in the clinic, asthma was suspected, however the prescribed inhaled corticosteroid and short acting $\beta 2$-agonist had little effect on his symptoms. A chest radiograph at this time was normal. His breathlessness increased and when he was seen in

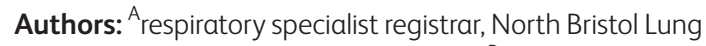
Centre, Southmead Hospital, Bristol, UK; ${ }^{\text {B }}$ respiratory specialist registrar, Academic Respiratory Unit, University of Bristol, Bristol, UK; ' Consultant histopathologist, Department of histopathology, University Hospitals Bristol, Bristol, UK; ${ }^{\text {D }}$ consultant respiratory physician, North Bristol Lung Centre, Southmead Hospital, Bristol, UK; ${ }^{E}$ consultant respiratory physician, Bristol Interstitial Lung Disease Service, North Bristol Lung Centre, Southmead Hospital, Bristol, UK

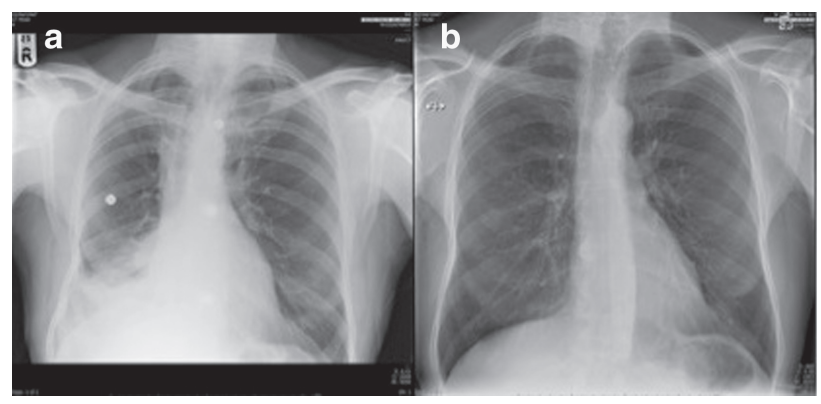

Fig 1. Chest radiographs (a) before treatment showing right middle lobe collapse and (b) after treatment showing resolution.

respiratory clinic he was struggling to walk 30 yards. He was expectorating brown cylindrical mucus plugs. Examination in clinic was consistent with right lower lobe collapse and oxygen saturations were $91 \%$ on air.

A chest radiograph in the clinic revealed right middle and lower lobe collapse (Fig 1) and a sputum sample grew Aspergillus fumigatus. A computed tomography of the patient's chest, abdomen and pelvis showed occlusion of the bronchus intermedius with soft tissue. There was no evidence of a mass lesion (Fig 2). Blood tests are shown in Table 1. Peripheral eosinophils were raised, as were total immunoglobulin (Ig) E, IgE to Aspergillus and IgG to Aspergillus.

Flexible bronchoscopy was performed, demonstrating a substantial, thick mucus plug in the bronchus intermedius (Fig 3). This was aspirated and a broncho-alvelolar lavage was undertaken. Endobronchial biopsies showed significant eosinophilia and there was no evidence of malignancy. Microscopy of the mucus plug showed branching Aspergillus hyphae (Fig 3) and Aspergillus fumigatus complex was cultured which was sensitive to voriconazole and itraconazole.

The patient was seen by the respiratory physiotherapists to teach sputum clearance techniques which led to partial reinflation of the right lower lobe; however this was not maintained so he was commenced on oral prednisolone and voriconazole. One month later a chest radiograph revealed persistent right lower lobe collapse. Repeat flexible bronchoscopy one month after treatment commenced again demonstrated mucus plugging within the medial segment of the right lower lobe. On this occasion, no fungus was cultured. 


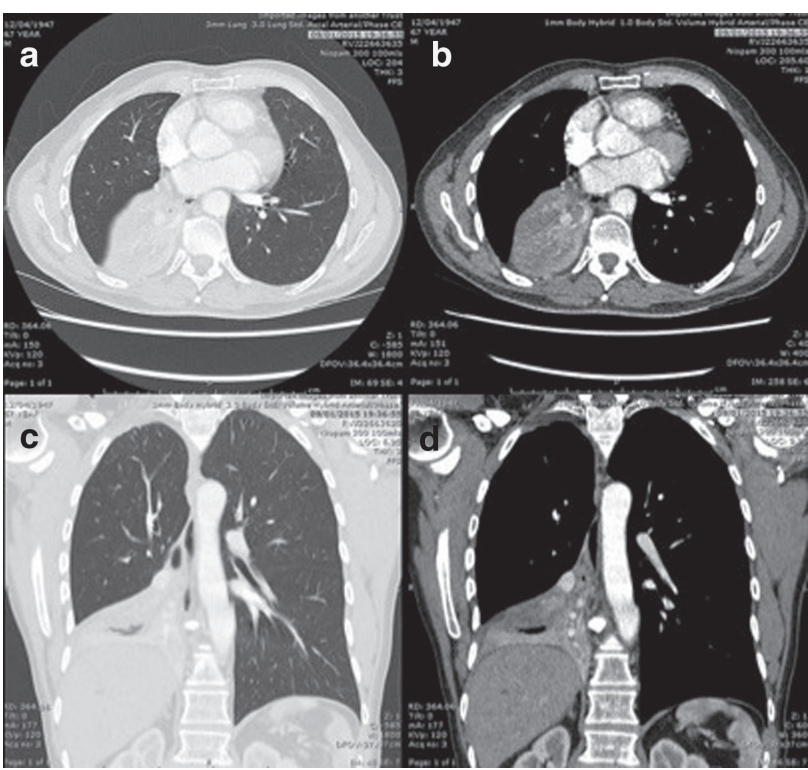

Fig 2. CT thorax showing right middle lobe collapse (sagittal and coronal views).

After two months of treatment, eosinophils had normalised and the right lower lobe collapse had completely resolved (Fig 1). The prednisolone dose was gradually weaned and he completed a three-month course of voriconazole.

\section{Discussion}

Aspergillus is ubiquitous in the environment; however only a minority of people go on to develop clinically significant disease. Aspergillus infection is an important cause of morbidity and mortality, and diagnosis is frequently missed or delayed. Risk factors for invasive Aspergillus infections are well recognised and include underlying respiratory disease, immunosupression, corticosteroid use and previous lung transplantation. ${ }^{1}$ It is less commonly seen in an immunocompetent patient with no underlying lung disease.

Cases have been reported of invasive Aspergillus disease following massive inhalation of Aspergillus spores, as in our case. Previously reported sources for massive inhalation of Aspergillus spores include mouldy hay, ${ }^{2}$ bark chippings ${ }^{3}$ and rotten tree stumps, ${ }^{4}$ mushroom pickers ${ }^{5}$ and following near drowning ${ }^{6}$.

With underlying immunosuppression, onset of disease is often insidious. Early detection and treatment is necessary to prevent permanent lung or airways damage. By contrast, immunocompetent subjects can have manifested features of invasive disease as soon as one day after massive environmental exposure to Aspergillus spores. ${ }^{6}$

In the literature, a spectrum of disease is reported. Allergic broncho-pulmonary aspergillosis (ABPA) ${ }^{7}$ and hypersensitivity pneumonitis to Aspergillus ${ }^{2}$ and invasive pulmonary Aspergillus disease $^{4}$ can all occur following inhalation of high levels of spores. There have been instances of fatal acute invasive pulmonary aspergillosis in immunocompetent hosts with no underlying respiratory disease following massive inhalation of fungal spores. ${ }^{5}$ Aspergillus tracheobronchitis is part of

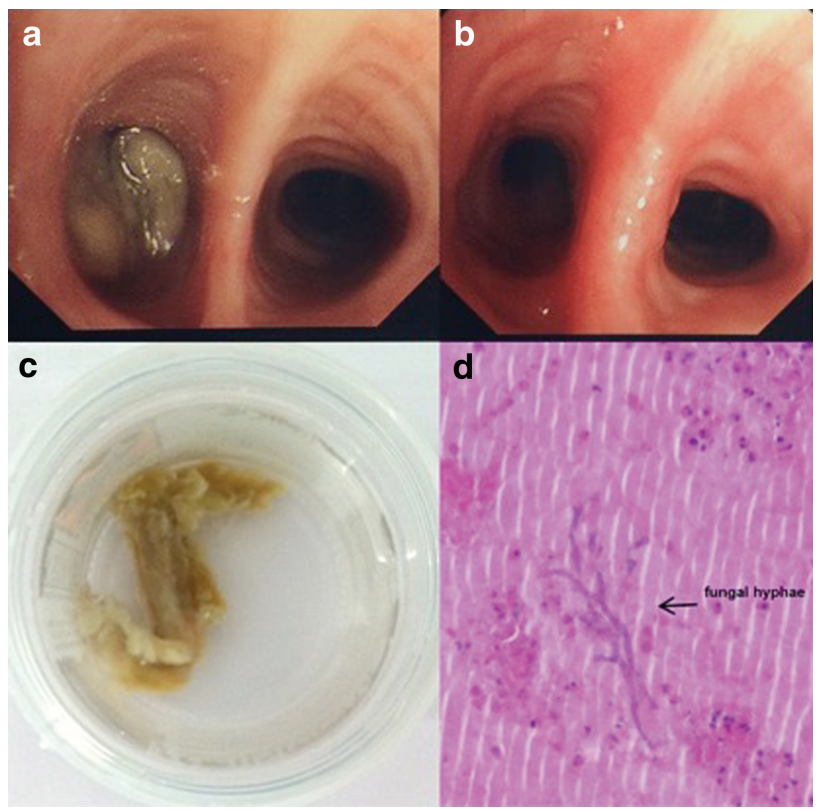

Fig 3. (a) Mucus plugging of right main bronchus at bronchoscopy; (b) right main bronchus post aspiration of mucus plug; (c) macroscopic view of mucus plug; and (d) histology showing branching of fungal hyphae.

Table 1. Summary of blood results at presentation.

\begin{tabular}{lll} 
Blood test & Result & Normal range \\
Haemoglobin & $142 \mathrm{~g} / \mathrm{L}$ & $130-170 \mathrm{~g} / \mathrm{L}$ \\
Mean corpuscle volume & $83.1 \mathrm{fL}$ & $80-100 \mathrm{fL}$ \\
Platelets & $301 \times 10^{9} / \mathrm{L}$ & $150-450 \times 10^{9} / \mathrm{L}$ \\
Leukocytes & $10 \times 10^{9} / \mathrm{L}$ & $4.5-6 \times 10^{9} / \mathrm{L}$ \\
Neutrophils & $4.88 \times 10^{9} / \mathrm{L}$ & $1.8-8 \times 10^{9} / \mathrm{L}$ \\
Lymphocytes & $2.28 \times 10^{9} / \mathrm{L}$ & $1-4 \times 10^{9} / \mathrm{L}$ \\
Monocytes & $0.84 \times 10^{9} / \mathrm{L}$ & $0.2-8 \times 10^{9} / \mathrm{L}$ \\
Eosinophils & $1.94 \times 10^{9} / \mathrm{L}$ & $<0.41 \times 10^{9} / \mathrm{L}$ \\
Sodium & $132 \mathrm{mmol} / \mathrm{L}$ & $133-146 \mathrm{mmol} / \mathrm{L}$ \\
Potassium & $4.4 \mathrm{mmol} / \mathrm{L}$ & $3.5-5.3 \mathrm{mmol} / \mathrm{L}$ \\
Urea & $6.5 \mathrm{mmol} / \mathrm{L}$ & $2.5-7.8 \mathrm{mmol} / \mathrm{L}$ \\
Creatinine & $90 \mu \mathrm{mol} / \mathrm{L}$ & $60-110 \mu \mathrm{mol} / \mathrm{L}$ \\
eGFR calculated MDRD & $73 \mathrm{~mL} / \mathrm{min} / 1.73 \mathrm{~m}^{2}$ & \\
Calcium (adjusted) & $2.39 \mathrm{mmol} / \mathrm{L}$ & $2.2-2.6 \mathrm{mmol} / \mathrm{L}$ \\
C-reactive protein & $2 \mathrm{mg} / \mathrm{L}$ & $<6 \mathrm{mg} / \mathrm{L}$ \\
Total IgE & $1,519 \mathrm{kU} / \mathrm{L}$ & $1-113 \mathrm{kU} / \mathrm{L}$ \\
Specific Aspergillus IgE & Grade $3(\mathrm{moderate}$ & $<0.35 \mathrm{kUA} / \mathrm{L}$ \\
& $+v e)(5.15 \mathrm{kUA} / \mathrm{L})$ & $<40 \mathrm{mg} / \mathrm{L}$ \\
\hline Aspergillus IgG & $>200 \mathrm{mg} / \mathrm{L}$ & \\
\hline
\end{tabular}

eGFR = estimated glomerular filtration rate; $\mathrm{Ig}=$ immunoglobulin; $\mathrm{MDRD}=$ modification of diet in renal disease formula. 
the spectrum of invasive Aspergillus disease. Infection is predominantly contained within the airways and does not invade into the lung parenchyma.

Accurate diagnosis is needed due to the contrasting treatments for these conditions. Immunosuppression with corticosteroids is the mainstay of treatment for ABPA and hypersensitivity pneumonitis, which would significantly worsen invasive aspergillosis. Diagnosis of invasive aspergillosis is made by a combination of identification of Aspergillus fungus either in sputum or broncho-alveolar lavage, and consistent radiological features and serological testing. A high clinical index of suspicion is needed.

Voriconazole is the antifungal of choice for invasive Aspergillus however this is contraindicated in liver failure. In these instances, amphotericin B, caspofungin or micafungin are alternatives. Duration of treatment depends on clinical response but is typically for at least three months.

\section{Learning points}

> Invasive Aspergillus is rare in immunocompetent hosts; however it can present acutely especially after massive inhalation of fungal spores. There can be a range of presentations.

$>$ A high clinical index of suspicion is needed, and diagnosis is based on isolating the fungus, consistent radiology and serological testing.

$>$ Voriconazole is the antifungal of choice for invasive Aspergillus.

\section{Acknowledgements}

The authors would like to thank Dr Andrew Medford for his help with the title and reading of the manuscript.

\section{References}

1 Kosmidis C, Denning DW. The clinical spectrum of pulmonary aspergillosis. Thorax 2015;70:270-7.

2 Meeker DP, Gephardt GN, Cordasco EM Jr et al. Hypersensitivity pneumonitis versus invasive pulmonary aspergillosis: two cases with unusual pathologic findings and review of the literature. Am Rev Respir Dis 1991;143:431-6.

3 Butler L, Brockley T, Denning D et al. Acute Aspergillus pneumonia associated with mouldy tree bark-chippings, complicated by antiglomerular basement membrane disease causing permanent renal failure. Med Mycol Case Rep 2013;2:125-7.

4 Arendrup MC, O'Driscoll B R, Petersen E et al. Acute pulmonary aspergillosis in immunocompetent subjects after exposure to bark chippings. Scand J Infect Dis 2006;38:945-9.

5 Shimaoka Y, Ishida T, Kawasaki S et al. An autopsy case of Aspergillus pneumonia after inhalation of a great deal of dust in a mushroom factory. J Jpn Resp Soc 2006;44:659-64, in Japanese.

6 Ratermann KL, Ereshefsky BJ, Fleishaker EL et al. Fulminant invasive pulmonary aspergillosis after a near-drowning accident in an immunocompetent patient. Ann Pharmacother 2014;48:1225-9.

7 Bavishi A, Berman A. Aspergillus bronchitis causing total lung collapse in an immunocompetent patient. Chest 2013;144:206A-A.

Address for correspondence: Dr H Adamali, BILD Service, North Bristol Lung Centre, Southmead Hospital, Bristol BS10 5NB, UK. Email: huzaifa.adamali@nbt.nht.uk

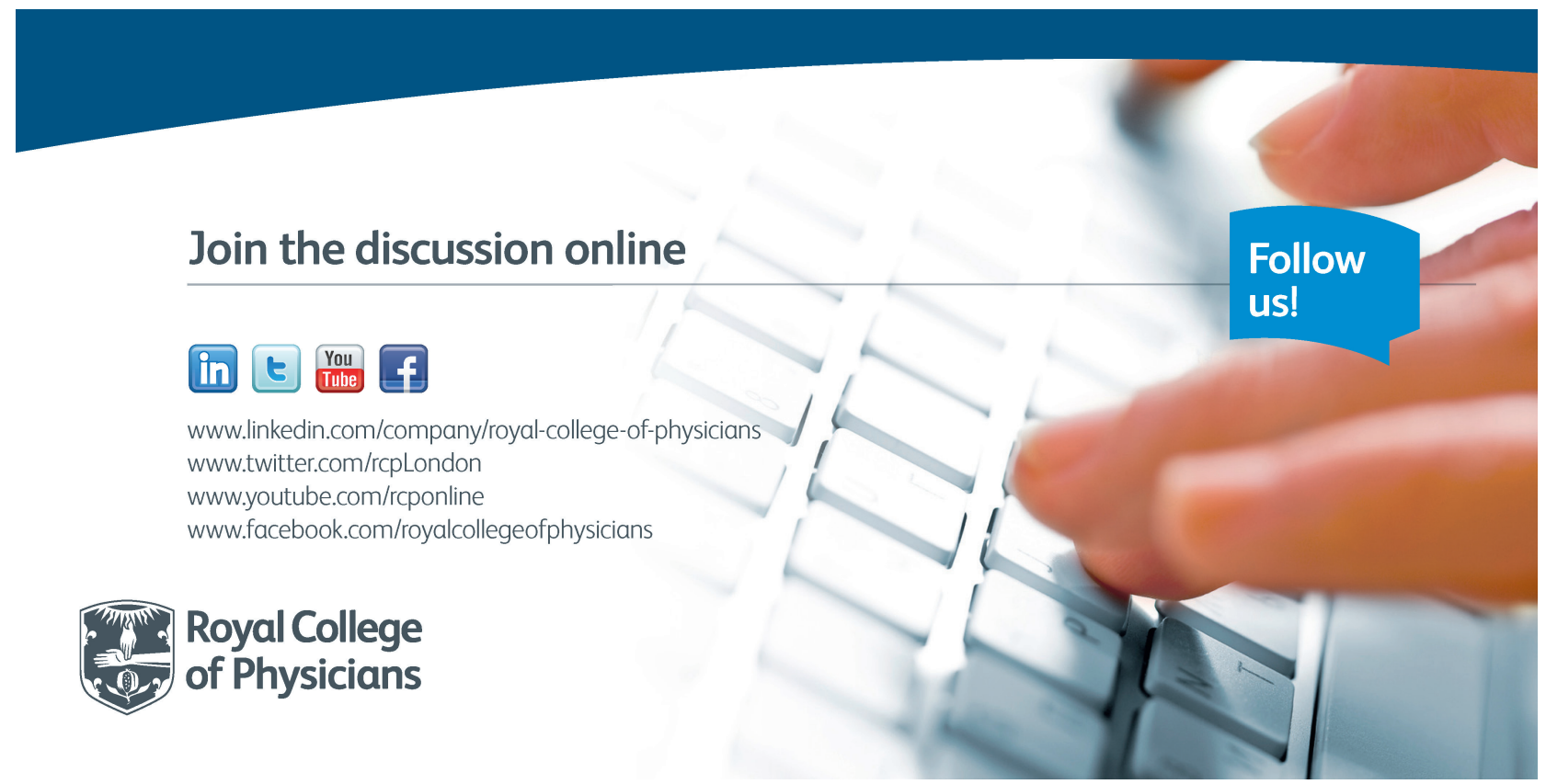

